\title{
The value of endoscopic ultrasound after bronchoscopy to diagnose thoracic sarcoidosis
}

\author{
K.G. Tournoy, A. Bolly, J.G. Aerts, P. Pierard, R. De Pauw, D. Leduc, A. Leloup, \\ T. Pieters, H. Slabbynck, A. Janssens, K. Carron, L. Schrevens, K. Pat, \\ T. De Keukeleire and C. Dooms
}

ABSTRACT: A clinicoradiological presentation of thoracic sarcoidosis requires histopathology in order to establish the diagnosis. Flexible bronchoscopy has a reasonable diagnostic yield and is the procedure of first choice for diagnosis. Endoscopic ultrasound (endoscopic ultrasoundguided fine needle aspiration/endobronchial ultrasound-guided transbronchial needle aspiration) can help in the diagnosis of sarcoidosis.

An implementation strategy of endoscopic ultrasound for the diagnosis of sarcoidosis following negative flexible bronchoscopy results was examined prospectively in 15 clinics.

A total of 137 patients (92 males; median age $43 \mathrm{yrs}$ ) were included, and sarcoidosis was found in 115 (84\%). Alternative diagnoses were tuberculosis, lymphangitis carcinomatosa, pneumoconiosis and alveolitis. All patients were sent for flexible bronchoscopy, which was performed in 121 $(88 \%)$, resulting in a definite diagnosis in $57(42 \%)$. A total of 80 patients were sent for endoscopic ultrasound, which could be performed in $72(90 \%)$, yielding a definite diagnosis in $47(59 \%)$. Endoscopic ultrasound following negative flexible bronchoscopy avoided a surgical procedure in 47 out of 80 patients. The sensitivity of flexible bronchoscopy for sarcoidosis was $45 \%(95 \%$ confidence interval $35-54 \%)$, but $62 \%(50-72 \%)$ if biopsy specimens were taken. The sensitivity of endoscopic ultrasound following negative flexible bronchoscopy results was $71 \%(58-82 \%)$. With this strategy, 97 out of $115(84 \%(76-90 \%))$ of proven sarcoidosis was diagnosed using endoscopy.

This large prospective implementation study (trial number NCT00888212; ClinicalTrials.gov) shows that endoscopic ultrasound is valuable for diagnosing sarcoidosis after negative flexible bronchoscopy results.

KEYWORDS: Bronchoscopy, endobronchial ultrasound-guided transbronchial needle aspiration, endoscopic ultrasound, endoscopic ultrasound-guided fine needle aspiration, mediastinoscopy, sarcoidosis

S arcoidosis is a disease of unknown aetiology and affects $5-40$ per 100,000 population, making it the most prevalent interstitial lung disease in the Western world [1]. There is no single diagnostic test. Its diagnosis is based on a compatible clinical and/ or radiological picture, supported by pathological evidence of noncaseating epithelioid cell granulomas in the absence of organisms or particles [1]. The diagnosis of sarcoidosis is reasonably certain without biopsy only in patients with Löfgren's syndrome. Otherwise, a biopsy specimen should be obtained from an involved organ that is most easily accessed. Since pulmonary sarcoidosis is the most frequent form, bronchoscopy with tissue sampling is advised as the first step in obtaining a tissue-based diagnosis and to exclude possible alternative diagnoses. Flexible bronchoscopy is cheap and has a reasonable diagnostic yield, especially if transbronchial biopsy (TBB) specimens are taken [2]. However, clinicians are frequently confronted with a nondiagnostic result following bronchoscopy, requiring a decision to be made as to whether surgical biopsy should be performed. Cervical mediastinoscopy, and, in some cases, video-assisted thoracoscopic surgery (VATS) procedures are regarded as the next diagnostic step following nondiagnostic bronchoscopy. Although surgery has a high yield [3, 4], it requires general anaesthesia, is costly, is associated with a certain morbidity [5] and invariably results in scars.

\section{AFFILIATIONS}

For affiliations, please see the Acknowledgements section

CORRESPONDENCE

K.G. Tournoy

Dept of Respiratory Medicine, Building 7K12 I.E. Ghent University Hospital De Pintelaan 185 9000 Ghent Belgium E-mail: kurt.tournoy@UGent.be

Received: July 152009 Accepted after revision: Oct 182009 First published online: Nov 062009 
Recent reports show that, with either curvilinear transoesophageal endoscopic ultrasound (EUS) with fine-needle aspiration (FNA) (EUS-FNA) or endobronchial ultrasound (EBUS) with transbronchial needle aspiration (TBNA) (EBUS-TBNA), together referred to hereafter as E(B)US, noncaseating granulomas can be demonstrated upon sampling of the intrathoracic nodes and that, as such, these techniques can contribute to the diagnosis of sarcoidosis [6-10]. Although these reports indicate feasibility, none of them assessed the value of these techniques in routine daily practice, where bronchoscopy remains the first diagnostic step [1]. In addition, these reports invariably originate from tertiary care institutions, where E(B)US was performed by experts in the field, possibly resulting in an overestimation of the yield.

The present article reports on the largest prospective implementation trial, in which patients with suspected thoracic sarcoidosis in 15 hospitals were first sent for conventional bronchoscopy. Only if no definite diagnosis were obtained was the patient offered E(B)US (either EUS-FNA or EBUS-TBNA). It was hypothesised that the addition of $\mathrm{E}(\mathrm{B}) \mathrm{US}$, after preceding nondiagnostic bronchoscopy, would result in an increase in diagnostic yield and that, using this strategy, the need for surgical biopsy could be reduced in a significant number of patients.

\section{METHODS}

\section{Study design and patients}

The study was performed in 15 participating hospitals, where consecutive patients with a clinicoradiological suspicion of thoracic sarcoidosis were included between June 2008 and May 2009. The study was approved by the central ethics committee of Ghent University Hospital (Ghent, Belgium; UZG2008069) and all 14 local committees. The protocol was registered at ClinicalTrials.gov (trial number NCT00888212) under the acronym MITOSIS (Minimally Invasive Techniques Or Surgery In Sarcoidosis). The study was designed as a prospective implementation study. The 15 participating chest physicians (median age $40 \mathrm{yrs}$; range 32-61 yrs) were all experienced with routine bronchoscopy procedures, and $80 \%$ were involved in respiratory training programmes (including bronchoscopy). Eleven performed E(B)US independently, and four referred their patients to one of the participating colleagues where needed. The median annual number of $\mathrm{E}(\mathrm{B}) \mathrm{US}$ procedures was 120 (range 30-250). The median time in practice was 7 (range 2-31) yrs. Written informed consent was obtained from all patients. Consecutive patients with suspected sarcoidosis in whom tissue confirmation was considered necessary were recruited. Patients with other than lung involvement, where simple diagnostic biopsy could be performed to obtain the diagnosis, were excluded. Accordingly, patients with Löfgren's syndrome, patients unfit to undergo endoscopy or a surgical intervention, and patients unable to provide written informed consent were excluded from participation in the trial.

The participating chest physicians were instructed to follow a diagnostic algorithm developed to measure the yield of bronchoscopy and E(B)US in case bronchoscopy did not result in a definite diagnosis. If no definite diagnosis was obtained following E(B)US, surgical biopsy was proposed. Samples were analysed according to the institutional practices. Rapid on-site analysis was not available. The presence of naked granulomas in lung biopsy specimens or noncaseating granulomas and giant cells on FNA or biopsy of the lymph nodes was considered sufficient for making the diagnosis of sarcoidosis in this population. If the samples were not representative or if they showed normal tissue, the result was categorised as nondiagnostic.

\section{Bronchoscopy}

Flexible bronchoscopy was the first step in the diagnostic algorithm. The procedure was performed according to institutional practice. Since data have shown the benefit of routinely adding endobronchial biopsy (EBB) and TBNA to traditional $\mathrm{TBB}$, the endoscopists were encouraged to apply these procedures during bronchoscopy [2]. Bronchoalveolar washing was routinely performed for microbiological analysis, whereas performance of bronchoalveolar lavage was left to the discretion of the local endoscopist. All procedural details were recorded, as were complications.

\section{Endoscopic ultrasound}

EBUS-TBNA and/or EUS-FNA were performed in the participating centres. The choice as to which technique to perform depended upon the investigators' preference and the availability of the technique. EBUS-TBNA and EUS-FNA were performed as outpatient procedures, using a curvilinear scanning ultrasound bronchoscope (BF UC160F OL8; Olympus, Aartselaar, Belgium) connected to an ultrasound unit (EU-C60; Olympus, or $\alpha 5-10 ;$ ALOKA, Mechelen, Belgium) and a curvilinear scanning oesophagoscope (GFUCT160-OL5; Olympus) connected to the ultrasound unit (ALOKA). The procedures were performed under local anaesthesia and moderate sedation or general anaesthesia according to the investigators' preference. Both EBUS-TBNA (NA-2015X-4022; Olympus) and EUS-FNA (NA-200H-8022; Olympus) were performed using a 22-gauge needle. Patients were observed for $2 \mathrm{~h}$ following the procedure. Cell smears of aspirates were stained using a quick staining method (DiffQuick ; Medion Diagnostics, Düdingen, Switzerland), whereas cell suspensions were collected in CytoRich ${ }_{\circledast}$ medium (Tripath Imaging, Inc., Burlington, NC, USA) for carrying out Papanicolaou's staining and making paraffin cell blocks.

\section{Surgical procedures}

Only if a preceding bronchoscopy and E(B)US procedure did not result in a definite diagnosis was the patient referred for surgical biopsy. The type of intervention was guided by the clinicoradiological presentation. Primarily, mediastinoscopy was advised in the case of unexplained mediastinal or hilar lymphadenopathies, although a VATS procedure with parenchymal biopsy was permitted if thought necessary.

\section{Sample size and data analysis}

Standardised evaluation forms for recording demographic characteristics, technical investigations and procedural characteristics were available to all investigators. All data were transferred into an electronic database (SPSS 17.0; SPSS, Inc., Chicago, IL, USA). The co-primary end-points of the study were the sensitivity of bronchoscopy and $\mathrm{E}(\mathrm{B}) \mathrm{US}$ in diagnosing sarcoidosis following a negative bronchoscopy result. In order to demonstrate a gain of $15 \%$ diagnostic yield by $\mathrm{E}(\mathrm{B}) \mathrm{US}$ 
following negative bronchoscopy results, the latter having a yield of $60 \%$, it was calculated that, with a type 1 error of $5 \%$ and a power of $90 \%$, a sample size for one proportion of 104 patients with sarcoidosis would be needed. Taking into account the fact that $80 \%$ of all patients would indeed end up with sarcoidosis, the aim was to collect data from 130 patients. Data were analysed according to the intention-todiagnose principle unless explicitly indicated (per-protocol analysis). Secondary end-points were technical characteristics, complication rates, protocol adherence and multivariate analysis to find determining factors. Diagnostic yields were compared using Fisher's exact test (two-sided).

\section{RESULTS}

\section{Patient characteristics}

In table 1, the main demographic characteristics of the 137 Caucasian patients are summarised. There were twice as many males as females, and approximately two-thirds were neversmokers. On chest radiography, $\sim 60 \%$ were classified as stage $0-I$, whereas $40 \%$ were thought be of stage II-IV. All patients underwent computed tomography, and the majority had enlarged (largest short axis of $\geqslant 10 \mathrm{~mm}$ ) lymph nodes, whereas parenchymal abnormalities were found in $52 \%$.

\section{Procedures and diagnoses}

The procedures performed are summarised in a flow chart (fig. 1). Bronchoscopy was performed in 121 (88\%) patients, and resulted in a definite diagnosis in $57(42 \%)$. In $16(12 \%)$ patients, bronchoscopy was cancelled. A total of $80(58 \%)$ patients had no definite diagnosis after bronchoscopy and were, therefore, scheduled for an E(B)US procedure. Either EUS-FNA or EBUS-TBNA was performed in 72 (90\%) patients, resulting in a definite diagnosis in 47 (59\%). The cancellation of

TABLE 1 Demographic characteristics of the study
population

Data are presented as $\mathrm{n}(\%)$ or mean (95\% confidence interval) unless otherwise indicated. \% pred: \% predicted; TLC: total lung capacity; VC: vital capacity; FEV1: forced expiratory volume in $1 \mathrm{~s}$; $D \mathrm{~L}, \mathrm{CO}$ : diffusing capacity of the lung for carbon monoxide; CT: computed tomography; LN: lymph node; ILD interstitial lung disease. ${ }^{\# \text { : }}$ using body plethysmography.

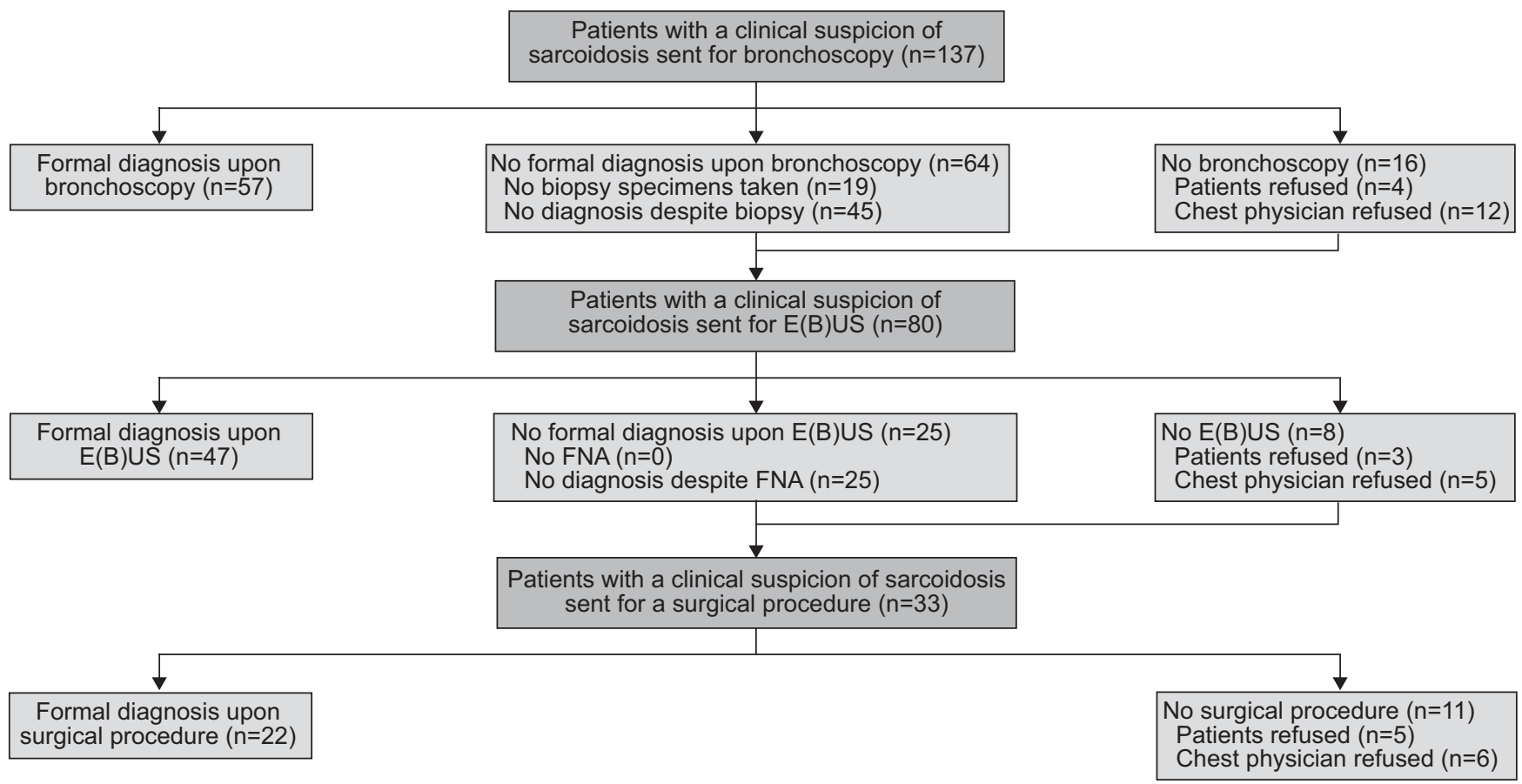

FIGURE 1. Procedures performed in the study population. E(B)US: endoscopic ultrasound in the form of either transoesophageal endoscopic ultrasound-guided fine needle aspiration (FNA) or endobronchial ultrasound-guided transbronchial needle aspiration. 


\begin{tabular}{ll} 
TABLE 2 & $\begin{array}{l}\text { Final pathological diagnosis of the mediastinal } \\
\text { lymph nodes in the study population }\end{array}$ \\
Sarcoidosis & $115(84)$ \\
Tuberculosis & $5(4)$ \\
Other diseases & $6(4)$ \\
No definite pathological diagnosis & $11(8)$ \\
\hline
\end{tabular}

Data are presented as $n$ (\%). Other diseases included extrinsic allergic alveolitis $(n=2)$, lymphangitis carcinomatosa $(n=1)$, pneumoconiosis $(n=1)$ and aspecific lymphadenitis shown by surgical biopsy $(n=2)$. No definite pathological diagnosis was obtained when bronchoscopy or endobronchial/ endoscopic ultrasound did not yield a diagnosis differing from that of unrepresentative or benign lymphadenitis, and which was not confirmed by surgical biopsy.

planned bronchoscopy or E(B)US in 16 and eight patients, respectively, was related to a variety of reasons, including refusals upon second thoughts, technical failures and endoscopists judging bronchoscopy or $\mathrm{E}(\mathrm{B}) \mathrm{US}$ to have a very unfavourable risk/benefit ratio in particular cases. As a result, $33(24 \%)$ patients were left without a definite diagnosis after bronchoscopy and E(B)US; 22 underwent a surgical procedure that resulted in a definite diagnosis. In 11 patients, surgical intervention was refused by the patient, or was thought to have no added value over a follow-up strategy.

With the current implementation strategy, a definite histopathological diagnosis was obtained in 126 (92\%) patients of

\begin{tabular}{lc} 
TABLE 3 Procedural details of bronchoscopy \\
Subjects $\mathbf{n}$ \\
$\begin{array}{l}\text { Anaesthesia } \\
\text { Local }\end{array}$ \\
Local plus sedation & 121 \\
General & $91(75)$ \\
Biopsy method & $30(25)$ \\
TBNA & $0(0)$ \\
EBB & \\
TBB & $26(21)$ \\
No biopsy taken & $76(63)$ \\
Diagnostic procedure & $76(63)$ \\
TBNA & $19(16)$ \\
EBB & \\
TBB & $8(14)$ \\
Wash and microbiology & $15(26)$ \\
Complications & $41(72)$ \\
Bleeding (minor) & $2(4)$ \\
Intolerance and stop & \\
Pneumothorax & $5(4)$ \\
Other & $3(2)$ \\
\hline
\end{tabular}

Data are presented as $n(\%)$ unless otherwise indicated. TBNA: transbronchial needle aspiration; EBB: endobronchial biopsy; TBB: transbronchial biopsy. " : a definite diagnosis (including sarcoidosis and other diagnoses) was obtained by bronchoscopy in 57 patients; this was by means of a unique method in 49 patients, whereas, in eight, a combination of methods was used. the study population. The final diagnoses are summarised in table 2. There were $115(84 \%)$ patients with sarcoidosis, whereas acid-fast bacilli were found in five. In six other patients, pneumoconiosis, alveolitis, lymphangitis carcinomatosa and aspecific lymphadenitis were found.

As summarised in table 3, bronchoscopy was performed mainly under local anaesthesia, although mild sedation was added in a quarter of procedures. TBNA was applied in $21 \%$, whereas EBB was performed in $63 \%$, as was TBB. In 19 patients, the endoscopist decided not to take a tissue sample except for a washing for microbiological and cytological analysis. A definite diagnosis was obtained by bronchoscopy in 57 patients. The majority of these diagnoses were obtained with TBB $(72 \%)$, which did better than EBB $(26 \%$; $\mathrm{p}=0.0003)$ even though both procedures were performed equally. The relative yield for all diagnoses per biopsy method was highest for TBB (41 diagnoses in 76 TBB procedures, or 54\%), followed by TBNA (31\%) and EBB (20\%). Minor complications were encountered with bronchoscopy: minor bleeding, intolerance, and one pneumothorax, the latter being treated with simple manual aspiration.

Table 4 shows the procedural characteristics of EUS-FNA and EBUS-TBNA. The majority of procedures were performed under local anaesthesia with mild sedation, whereas general anaesthesia was applied in 10\%. EBUS-TBNA was performed three times more than EUS-FNA. Mediastinal lymph nodes were sampled in 95\%, whereas hilar lymph nodes alone were approached in only four patients. The yield of EUS-FNA was $94 \%$ as compared to $56 \%$ for EBUS-TBNA ( $p=0.03)$. No

TABLE 4 Procedural details of endoscopic ultrasound
(E(B)US)

Data are presented as $n(\%)$ unless otherwise indicated. EUS-FNA: endoscopic ultrasound-guided fine needle aspiration; EBUS-TBNA: endobronchial ultrasound-guided transbronchial needle aspiration; LN: Iymph node. \#: the chance of obtaining a definite diagnosis (including sarcoidosis and other diagnoses) was significantly higher with EUS-FNA than EBUS-TBNA ( $p=0.03$; Fisher's exact test) 


\begin{tabular}{|c|c|c|c|}
\hline \multirow[t]{2}{*}{ TABLE 5} & \multicolumn{3}{|c|}{$\begin{array}{l}\text { Test characteristics of bronchoscopy and } \\
\text { endoscopic ultrasound (E(B)US) for making the } \\
\text { diagnosis of sarcoidosis }\end{array}$} \\
\hline & & $\begin{array}{c}\text { Diagnoses } \\
\mathbf{n}\end{array}$ & $\begin{array}{l}\text { Diagnoses } \\
\%(95 \% \mathrm{Cl})\end{array}$ \\
\hline \multicolumn{4}{|c|}{ Yield of bronchoscopy } \\
\hline Intention to & liagnose & $52 / 115$ & $45(35-94)$ \\
\hline Per protocc & & $52 / 100$ & $52(41-62)$ \\
\hline Per protocc & with biopsy" & $52 / 84$ & $62(50-72)$ \\
\hline \multicolumn{4}{|c|}{ Yield of $E(B) U S$} \\
\hline Intention to & liagnose & $45 / 63$ & $71(58-82)$ \\
\hline Per protocc & overall $^{+}$ & $45 / 58$ & $77(64-87)$ \\
\hline Per protocc & EUS $S^{\S}$ & $16 / 17$ & $94(91-99)$ \\
\hline Per protocc & EBUS ${ }^{f}$ & $29 / 41$ & $71(54-83)$ \\
\hline $\begin{array}{l}\text { Per protoce } \\
\text { bronchose }\end{array}$ & $\begin{array}{l}\text { after negative } \\
\text { oy } y^{\# \#}\end{array}$ & $33 / 43$ & $77(61-88)$ \\
\hline \multicolumn{4}{|c|}{ Yield of bronchoscopy plus E(B)US } \\
\hline Intention to & tiagnose & $97 / 115$ & $84(76-90)$ \\
\hline \multicolumn{4}{|c|}{ 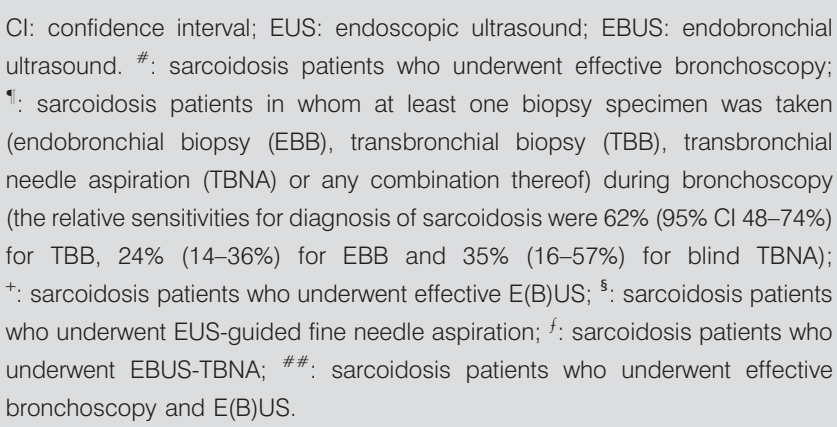 } \\
\hline
\end{tabular}

complications were noted in the patients investigated using E(B)US.

The surgical interventions performed in 22 patients were cervical mediastinoscopy in $19(86 \%)$, VATS in two $(9 \%)$ and open lung biopsy in one (5\%). All surgical procedures resulted in a definite diagnosis. One patient developed mediastinitis and was treated with antibiotics, with a favourable course.

\section{Test characteristics for diagnosing sarcoidosis}

When proposing bronchoscopy as a first diagnostic step, 52 $(45 \%)$ of the 115 patients who finally had sarcoidosis were identified. This diagnostic yield increased to 52 and $62 \%$, respectively, when bronchoscopy was performed effectively and when at least one biopsy specimen was taken (table 5). It was found that the sensitivity of finding sarcoidosis with bronchoscopy was $70 \%$ among females, but only $44 \%$ in males $(p=0.03)$. Not surprisingly, taking a biopsy specimen strongly determined the procedure to be successful $(p<0.0001)$.

With E(B)US proposed to the 63 remaining patients with sarcoidosis, the diagnosis was found in 45 (71\%). This diagnostic yield increased to $77 \%$ if the procedure were performed effectively. The diagnostic yield for sarcoidosis was comparable for EUS-FNA and EBUS-TBNA $(p=0.08)$. In the 20 patients with sarcoidosis investigated first with blind TBNA followed by E(B)US if necessary, the former yielded the diagnosis in eight $(40 \%)$, whereas addition of the latter increased the yield to $70 \%$. For E(B)US, it was found that none of the measured factors were predictive.

The overall diagnostic yield for sarcoidosis, with the proposed endoscopic strategy of bronchoscopy plus E(B)US in case the former is inconclusive, is $84 \%$. The incremental yield of adding $\mathrm{E}$ (B)US on to bronchoscopy is, therefore, 39\%. This means that, by adding $\mathrm{E}(\mathrm{B}) \mathrm{US}$ to prior nondiagnostic bronchoscopy, three patients should be investigated in order to avoid one surgical diagnostic procedure.

\section{DISCUSSION}

The most important finding of the present largest implementation trial ever on sarcoidosis is that, in patients with thoracic sarcoidosis, the proposed algorithm of bronchoscopy followed by $\mathrm{E}(\mathrm{B}) \mathrm{US}$ only in those cases in whom no definite diagnosis was obtained yields histological proof of the disease in $84 \%$. Although $45 \%$ of the sarcoidosis patients were diagnosed with bronchoscopy, $\mathrm{E}(\mathrm{B}) \mathrm{US}$ provided the diagnosis in an additional $39 \%$ of cases.

According to the available guidelines of the ERS, ATS and World Association of Sarcoidosis and Other Granulomatous Disorders, bronchoscopy should be the first diagnostic step [11]. Flexible bronchoscopy is a readily available, safe and well-tolerated procedure permitting several modalities of tissue sampling from different anatomical sites [2, 12]. TBB has been suggested to be the method of preference, with yields ranging $40-90 \%$ in case series [13-15]. EBB has added value, even without apparent endobronchial abnormalities [16]. The same holds for blind TBNA, especially when added to TBB [17-19]. Besides these procedures, bronchial washing is recommended for microbiological analysis, while bronchoalveolar lavage with CD4/CD8 counting is variable and less sensitive [20].

In the present study, it was found that, when bronchoscopy is offered as a first-line tool in a study population with a suspicion of having thoracic sarcoidosis, the overall diagnostic yield is $42 \%$, and the sensitivity for sarcoidosis is $45 \%$. This is at the lower end of what has been reported previously [2], and illustrates the erosion of diagnostic yield once a technique is widely implemented. A possible reason for this is that all of the aforementioned studies were performed in expert centres, where specialised endoscopists investigated very selected patients. This differs greatly from implementation studies, where a variable degree of expertise is present and where some of the procedures were even omitted or only partially carried out because of a variety of patient, endoscopist or technical reasons. In addition, it requires a great deal of expertise to perform all biopsy modalities during a single bronchoscopy session. It is also remarkable to note that only 76 patients underwent TBB; the same number underwent $\mathrm{EBB}$ and 26 TBNA. This shows that endoscopists do not always feel comfortable performing some of the biopsy procedures. Nevertheless, when per-protocol analysis of the yield of TBB in the current study was performed, it was found that, in $72 \%$ of the procedures, a definite diagnosis was obtained. For sarcoidosis alone, the sensitivity of TBB was $62 \%$. This is a value comparable to those published before [12, 15]. As reported recently, there was also no relationship found between TBB yield and the stage of sarcoidosis on chest 
radiography [15]. The safety figures of bronchoscopy appeared very acceptable, with only one case of pneumothorax, which was cured with manual aspiration.

When bronchoscopy does not result in a definite diagnosis, and when no other accessible sites for biopsy are identified, the guidelines suggest that surgical biopsy may be indicated if there are readily identifiable radiological abnormalities [11]. The finding of mediastinal adenopathies should, therefore, prompt biopsy by mediastinoscopy before VATS or even open lung biopsy [4]. Although these procedures have a superior yield, they are costly and exhibit a comorbidity that cannot be denied. Given the above data obtained with bronchoscopy, it is clear that, in daily practice, new tools that could provide a definite diagnosis in a minimally invasive way are very welcome.

EUS-FNA and EBUS-TBNA are minimally invasive outpatient techniques that have shown good test characteristics in lung cancer staging [21, 22] and have been recommended in the guidelines [23, 24]. By consequence, these techniques are also being implemented in non-academic hospitals. To date, a few series have shown that EUS-FNA and EBUS-TBNA can be used to demonstrate sarcoidosis [6-10] in up to $80-90 \%$ of highly selected cases. However, what remains unknown is their value following a nondiagnostic bronchoscopy procedure.

We found that E(B)US following negative bronchoscopy provided a definite diagnosis in $59 \%$ of the patients in whom a surgical procedure would otherwise have been considered. The sensitivity for diagnosing sarcoidosis was $71 \%$. These figures are lower than those reported in the series in which EUS-FNA or EBUS-TBNA were evaluated mainly as a first-line tool for the diagnosis of sarcoidosis [6-10]. The reasons for this are probably comparable to the reasons discussed for the yield of bronchoscopy in an implementation setting. The present calculation also takes into account the fact that not all patients underwent effective E(B)US for a variety of reasons, such as refusal of a second endoscopic procedure. When applying the per-protocol analysis, a sensitivity of E(B)US of $77 \%$ for diagnosing sarcoidosis following negative bronchoscopy was found. Recently, it was shown that EBUS-TBNA had a 30\% higher yield for finding sarcoidosis than blind TBNA [25]. Although calculated in a subgroup, it was found that EBUSTBNA following false-negative blind TBNA also increased the yield by $30 \%$. This indicates that preceding negative blind TBNA is not a reason to skip $\mathrm{E}(\mathrm{B}) \mathrm{US}$ in patients with presumed sarcoidosis.

The overall strategy, in which patients with sarcoidosis are investigated first with bronchoscopy and then with E(B)US only if the former does not yield the diagnosis, results in an overall sensitivity of $84 \%$, representing an absolute increase of $39 \%$ above the yield of bronchoscopy. In the present study, the majority of the $\mathrm{E}(\mathrm{B}) \mathrm{US}$ procedures were performed under mild sedation, allowing the patient to leave the hospital after a short observation period. Although the safety of E(B)US has mainly been described in lung cancer populations, no serious complications were also noted in this series. We, therefore, conclude that the algorithm used here is valuable, safe and useful for daily practice.
Some points should be borne in mind when considering these data. First, a final histopathological diagnosis was pursued in all of the patients, although there were $11(8 \%)$ patients in whom this was not achieved. This figure is probably very reasonable taking into account the study population (mainly patients with benign diseases) and the fact this is an implementation trial. Nevertheless, it could be that there were patients with sarcoidosis that remained undetected. Secondly, it should be noted that the presence of noncaseating epithelioid granulomas without necrosis is not diagnostic per se for sarcoidosis. The diagnosis can only be made by an integration of the clinicoradiological picture, histological data and exclusion of other identifiable causes of granulomatous diseases [1, 11]. For example, sarcoid-like inflammation in lymph nodes near lymphomas or carcinomas [26], or in the context of histoplasmosis or tuberculosis, can be misdiagnosed as sarcoidosis. Conversely, histoplasmosis is virtually nonexistent in Northern Europe, and the currently applied methods proved to be sensitive for the diagnosis of mycobacterial disease, making the chance of misdiagnosis probably very low. Finally, the current data do not formally answer the question as to whether bronchoscopy should be continued or whether it is better to carry out or refer for immediate E(B)US when thoracic sarcoidosis is suspected. The only way of finding this out is to perform a randomised controlled trial with a direct comparison between the two. When thinking about this, it should, however, always be remembered that bronchoscopy is cheap and readily available because it is routinely taught to all chest physicians, which is not the case for E(B)US.

In conclusion, we propose a high-yield and safe diagnostic algorithm for patients with thoracic sarcoidosis requiring tissue confirmation, which states that they should first be investigated with bronchoscopy, followed by E(B)US only in those cases in whom no definite diagnosis is obtained.

\section{CLINICAL TRIAL}

This study is registered at ClinicalTrials.gov (trial number NCT00888212).

\section{STATEMENT OF INTEREST}

None declared.

\section{ACKNOWLEDGEMENTS}

The affiliations of the present authors are as follows. K.G. Tournoy: Dept of Respiratory Medicine, Ghent University Hospital, Ghent; A. Bolly: Dept of Respiratory Medicine, Clinique Sainte Elisabeth, Namur; P. Pierard: Dept of Respiratory Medicine, Centre Hospitalier Universitaire, Charleroi; R. De Pauw: Dept of Respiratory Medicine, Algemeen Ziekenhuis St Jan, Brugge; D. Leduc: Dept of Respiratory Medicine, Université Libre, Brussels; A. Leloup: Dept of Respiratory Medicine, Onze-Lieve-Vrouw Ziekenhuis, Aalst; T. Pieters: Dept of Respiratory Medicine, Université Catholique de Louvain, Brussels; $\mathrm{H}$. Slabbynck: Dept of Respiratory Medicine, Ziekenhuis Netwerk Antwerpen, Antwerp; A. Janssens: Dept of Respiratory Medicine, University Hospital, Antwerp; K. Carron: Dept of Respiratory Medicine, Heilig Hart Ziekenhuis, Menen; L. Schrevens: Dept of Respiratory Medicine, Heilig Hart Ziekenhuis, Tienen; K. Pat: Dept of Respiratory Medicine, Virga Jesse Ziekenhuis, Hasselt; T. De Keukeleire: Dept of Respiratory Medicine, University Hospital Brussels, Brussels; and C. Dooms: Dept of Respiratory Medicine, University Hospitals Leuven, Leuven (all Belgium). J.G. Aerts: Dept of Respiratory Medicine, Amphia Ziekenhuis, Breda, the Netherlands. 


\section{REFERENCES}

1 Iannuzzi MC, Rybicki BA, Teirstein AS. Sarcoidosis. N Engl J Med 2007; 357: 2153-2165.

2 Chapman JT, Mehta AC. Bronchoscopy in sarcoidosis: diagnostic and therapeutic interventions. Curr Opin Pulm Med 2003; 9: 402-407.

3 Porte H, Roumilhac D, Eraldi L, et al. The role of mediastinoscopy in the diagnosis of mediastinal lymphadenopathy. Eur J Cardiothorac Surg 1998; 13: 196-199.

4 Gossot D, Toledo L, Fritsch S, et al. Mediastinoscopy vs thoracoscopy for mediastinal biopsy. Results of a prospective nonrandomized study. Chest 1996; 110: 1328-1331.

5 Hammoud ZT, Anderson RC, Meyers BF, et al. The current role of mediastinoscopy in the evaluation of thoracic disease. J Thorac Cardiovasc Surg 1999; 118: 894-899.

6 Wildi SM, Judson MA, Fraig M, et al. Is endosonography guided fine needle aspiration (EUS-FNA) for sarcoidosis as good as we think? Thorax 2004; 59: 794-799.

7 Annema JT, Veselic M, Rabe KF. Endoscopic ultrasound-guided fine-needle aspiration for the diagnosis of sarcoidosis. Eur Respir J 2005; 25: 405-409.

8 Fritscher-Ravens A, Sriram PV, Topalidis T, et al. Diagnosing sarcoidosis using endosonography-guided fine-needle aspiration. Chest 2000; 118: 928-935.

9 Wong M, Yasufuku K, Nakajima T, et al. Endobronchial ultrasound: new insight for the diagnosis of sarcoidosis. Eur Respir J 2007; 29: 1182-1186.

10 Garwood S, Judson MA, Silvestri G, et al. Endobronchial ultrasound for the diagnosis of pulmonary sarcoidosis. Chest 2007; 132: 1298-1304.

11 Statement on sarcoidosis. Joint Statement of the American Thoracic Society (ATS), the European Respiratory Society (ERS) and the World Association of Sarcoidosis and Other Granulomatous Disorders (WASOG) adopted by the ATS Board of Directors and by the ERS Executive Committee, February 1999. Am J Respir Crit Care Med 1999; 160: 736-755.

12 Costabel U, Ohshimo S, Guzman J. Diagnosis of sarcoidosis. Curr Opin Pulm Med 2008; 14: 455-461.

13 Gilman MJ, Wang KP. Transbronchial lung biopsy in sarcoidosis. An approach to determine the optimal number of biopsies. Am Rev Respir Dis 1980; 122: 721-724.
14 Koonitz $\mathrm{CH}$, Joyner LR, Nelson RA. Transbronchial lung biopsy via the fiberoptic bronchoscope in sarcoidosis. Ann Intern Med 1976; 85: 64-66.

15 de Boer S, Milne DG, Zeng I, et al. Does CT scanning predict the likelihood of a positive transbronchial biopsy in sarcoidosis? Thorax 2009; 64: 436-439.

16 Shorr AF, Torrington KG, Hnatiuk OW. Endobronchial biopsy for sarcoidosis: a prospective study. Chest 2001; 120: 109-114.

17 Morales CF, Patefield AJ, Strollo PJ Jr, et al. Flexible transbronchial needle aspiration in the diagnosis of sarcoidosis. Chest 1994; 106 709-711.

18 Trisolini R, Agli LL, Cancellieri A, et al. The value of flexible transbronchial needle aspiration in the diagnosis of stage I sarcoidosis. Chest 2003; 124: 2126-2130.

19 Trisolini R, Tinelli C, Cancellieri A, et al. Transbronchial needle aspiration in sarcoidosis: yield and predictors of a positive aspirate. J Thorac Cardiovasc Surg 2008; 135: 837-842.

20 Kantrow SP, Meyer KC, Kidd P, et al. The CD4/CD8 ratio in BAL fluid is highly variable in sarcoidosis. Eur Respir J 1997; 10: 2716-2721.

21 Micames CG, McCrory DC, Pavey DA, et al. Endoscopic ultrasound-guided fine-needle aspiration for non-small cell lung cancer staging: a systematic review and metaanalysis. Chest 2007; 131: 539-548

22 Gu P, Zhao YZ, Jiang LY, et al. Endobronchial ultrasound-guided transbronchial needle aspiration for staging of lung cancer: a systematic review and meta-analysis. Eur J Cancer 2009; 45: 13891396.

23 Detterbeck FC, Jantz MA, Wallace MB, et al. Invasive mediastinal staging of lung cancer. ACCP evidence based clinical practice guidelines (2nd edition). Chest 2007; 132: 202S-220S.

24 De Leyn P, Lardinois D, Van Schil PE, et al. ESTS guidelines for preoperative lymph node staging for non-small cell lung cancer. Eur J Cardiothorac Surg 2007; 32: 1-8.

25 Tremblay A, Stather DR, Maceachern P, et al. A randomized controlled trial of standard vs endobronchial ultrasonographyguided transbronchial needle aspiration in patients with suspected sarcoidosis. Chest 2009; 136: 340-346.

26 Steinfort DP, Irving LB. Sarcoidal reactions in regional lymph nodes of patients with non-small cell lung cancer: incidence and implications for minimally invasive staging with endobronchial ultrasound. Lung Cancer 2009; 66: 305-308. 